\title{
Ulcerative colitis and lymphoproliferative disorder
}

\begin{abstract}
The lymphoma of the primary gastrointestinal tract (LGIP) is a rare form of presentation of gastrointestinal tract lymphomas. Inflammatory bowel disease and its base treatment are known risk factors for its appearance. We report a 56-year-old female patient with Ulcerative Colitis of eight years of evolution, treated only with mesalazine due to azathioprine intolerance. After a recent weight loss and abrupt change in the intestinal habit, a new coloncoscopy was performed showing a rectal ulcerative lesion with a depressed center, corresponding to a follicular non-Hodkin B lymphoma. The patient was treated with RCHOP chemotherapy (Rituximab cyclophosphamide doxorubicin vincristine prednisone) initially successful but pending re-evaluation after 4 cycles for finally disease remission.
\end{abstract}

Keywords: colitis, ulcerative, inflammatory bowel disease, lymphoma, large b-cell, diffuse
Volume 10 Issue 3 - 2019

\author{
Germán Santamaría Rodríguez, Álvaro \\ Morales Prado, Amaro Luna Morales, Carlos \\ Rosell Martí \\ Hospital Universitario de Puerto Real, Spain
}

Correspondence: Germán Santamaría Rodríguez, Hospital Universitario de Puerto real (CÁDIZ), Spain,

Email germansrv@gmail.com

Received: May 08, 2019 | Published: May 29, 2019

\section{Introduction/Background}

Ulcerative colitis, as well as intestinal inflammatory diseases in general is associated with a higher incidence of neoplasms, both due to the chronic inflammatory process and to many of the drugs used. Emphasize colon adenocarcinoma, cholangiocarcinoma (associated with $\mathrm{PBC}$ ), lymphomas or skin cancer.

The lymphoma of the primary gastrointestinal tract (LGIP) describes the lymphoma that originates in the gastrointestinal tract, representing only $5-10 \%$ of all lymphomas ${ }^{1-3}$ and up to $20 \%$ of extranodal lymphomas 4,5 .

This type of lymphoma, however, is infrequent, constituting only $1-4 \%$ of gastrointestinal neoplasms 5 . Studies have suggested that the incidence of LGIP has increased, ${ }^{4,7}$ a trend that could be explained by the presence of immunosuppression states, whether mediated by an acquired immunodeficiency syndrome (AIDS), by drugs in the context of a post-transplant or associated with diseases autoimmune diseases such as rheumatoid arthritis, systemic lupus erythematosus or celiac disease. ${ }^{4,8,9}$

Among the drugs that favor the development of lymphoma, thiopurine (azathioprine and 6-mercaptopurine) ${ }^{10,11}$ and, to a lesser extent, anti-TNF biological therapy. ${ }^{11}$

Although LGIP can compromise any region of the gastrointestinal tract, the most affected areas are the stomach, followed by the small intestine and the ileocecal region. ${ }^{13}$ Although the colorectal region can be compromised in up to $20 \%$ of LGIP4, ${ }^{13,14}$ primary colorectal lymphoma is very rare, constituting $<1 \%$ of neoplasms at this level. ${ }^{14}$

Similarly to what occurs in other areas of the gastrointestinal tract, primary colorectal lymphoma is mainly a non-Hodgkin B-cell lymphoma, with diffuse large B-cell lymphoma being the most frequent. It can also present as a follicular lymphoma, lymphoma associated with lymphoid tissue of the mucosa, lymphoma of mantle cells and lymphocytic small cell. ${ }^{15,16}$

\section{Case report}

We present the case of a 56year-old woman, No allergic medication reactions, smoker of 10cig/day, an abortion (negative for Antiphospholipid syndrome) and with ischemic cerebrovascular stroke in 2010 without aftermath. Ulcerative pancolitis of 8years of evolution, in treatment with 5' ASA (Mesalazine), with last complete colonoscopy previous year, showing ulcerative proctosigmoiditis with moderate activity and the rest of the surrounding quiescent mucosae.

She consulted for intestinal tract disorder consisting of 2-3 daily stools alternating with constipation in the last month, loss of about $7 \mathrm{~kg}$ of weight at that time and general malaise with abdominal discomfort. The examination revealed a palpable mass of about $4 \mathrm{~m}$ in the right lower quadrant, not displaceable, painless that the patient had gone unnoticed and apparently not adhered to deep planes.

\section{Lab test only Ca I 25 of $359 \mathrm{U} / \mathrm{ml}$ stood out}

Ultrasonography: an inflammatory mass-plastron in the right iliac fossa was found in the theoretical ileocecal area of about $4 \times 5 \mathrm{~cm}$ maximum diameter, as well as bilateral pleural effusion, minimal perihepatic and perisplenic free ascites and retroperitoneal adenopathies. Also compression of both ureters with bilateral hydronephrosis

Abdominal CT: a large retroperitoneal mass is observed that involves vascular structures with thickening and hypercaptation of rectum, cecum and terminal ileum walls, as well as multiple inguinal adenopathies, the largest in the left iliac fossa of $26 \mathrm{~mm}$. Before an unexpected polyadenopathic picture with associated polyserositis, the etiological approach was initiated: Thoracentesis was initially performed with extraction of pleural fluid compatible with transudate, normal ADA.

\section{Negative Mantoux}

We consulted with the Surgery team and exeresis of the larger inguinal lymphadenopathy was carried out for anatomopathological study.

Subsequently, a colonoscopy was performed where, at the first Houston valve, an ulcerated lesion with a depressed center was identified, which appeared to extend around $1 / 3$ of the circumference of the rectum, from which multiple biopsies were also taken. With the presumptive diagnosis of eventual disseminated rectal cancer in a patient with the known inflammatory disease, both samples were submitted for A.P study.

Also a TPE was taken out glandular involvement supra and, to a greater extent, infradiafrgamatics, highlighting an increase 
in retroperitoneal hyper metabolic density; as well as intestinal infiltration and right parotid doubtful. Probable splenic and medullary bone hyperreactivity with dubious right iliac focus. Moderate amount of normometabolic ascitic fluid.

Proposed Ann-Arbor Stadium IV (Figures I - I 0)

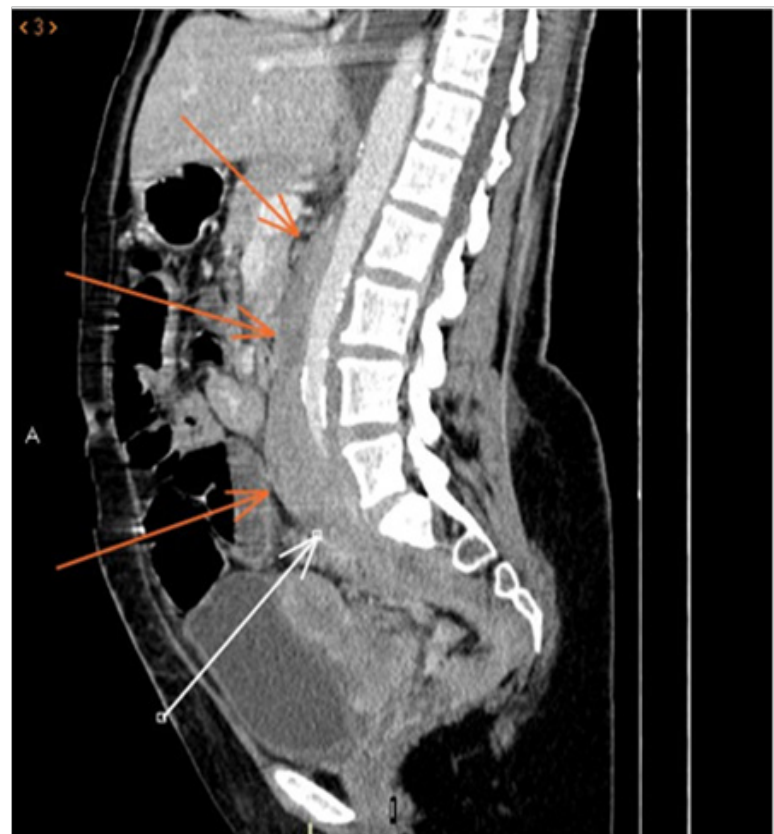

Figure I Sagital Scanner Tomography. Aortoiliac lymphadenopathies.

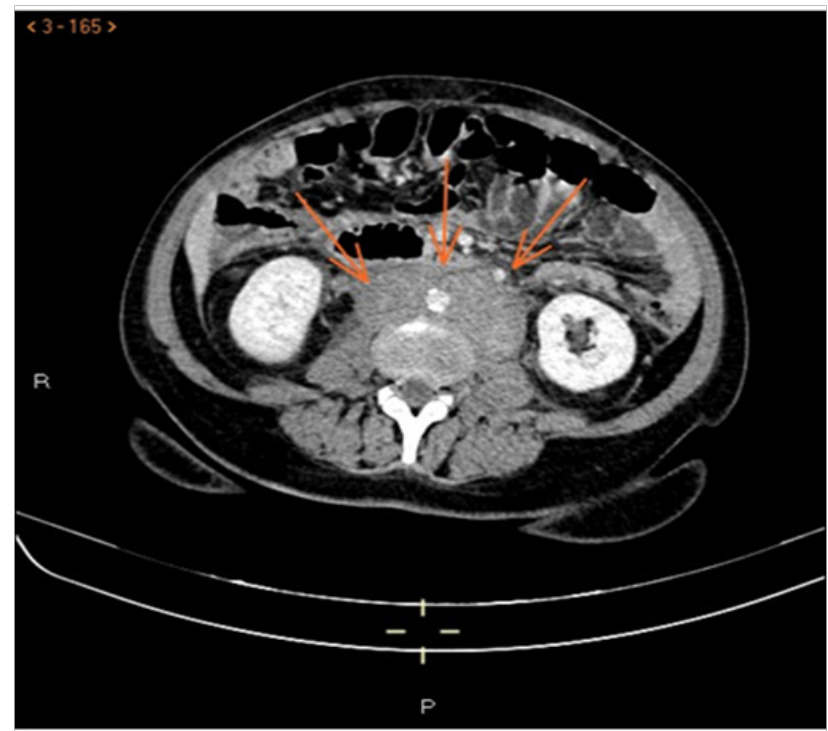

Figure 2 Axial Scanner Tomography. Retroperitoneal lymph nodes.

Finally, after histopathological and immunohistochemical study, the diagnosis of follicular non-Hodkin B lymphoma, histological grade 2, was confirmed in both anatomopathological samples. It was referred to Hematology for treatment with the diagnosis of NonHodgkin B Follicular Lymphoma Grade 2 Bulky abdominal. Stage IIIB. FLIPI 2, taking a therapeutic regimen with CHOP (Rituximab + Cyclophosphamide + Adriamycin + Vincristine and prednisone) with re-evaluation after 4 cycles.

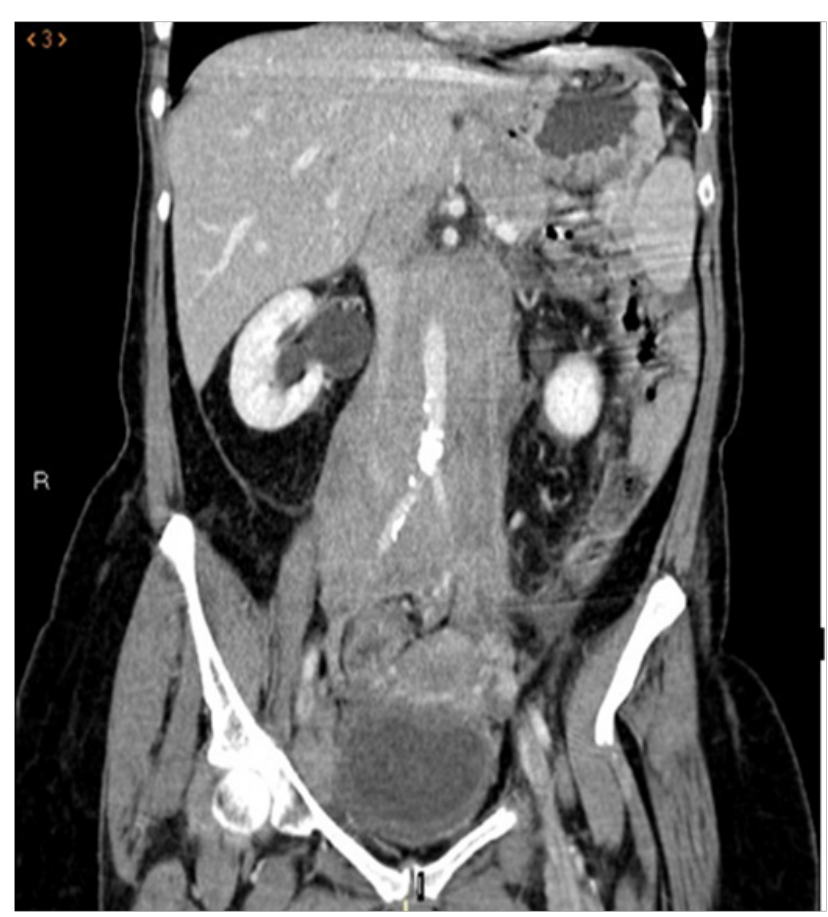

Figure 3 Coronal scanner. Polyadenopathic process involving vascular structures.

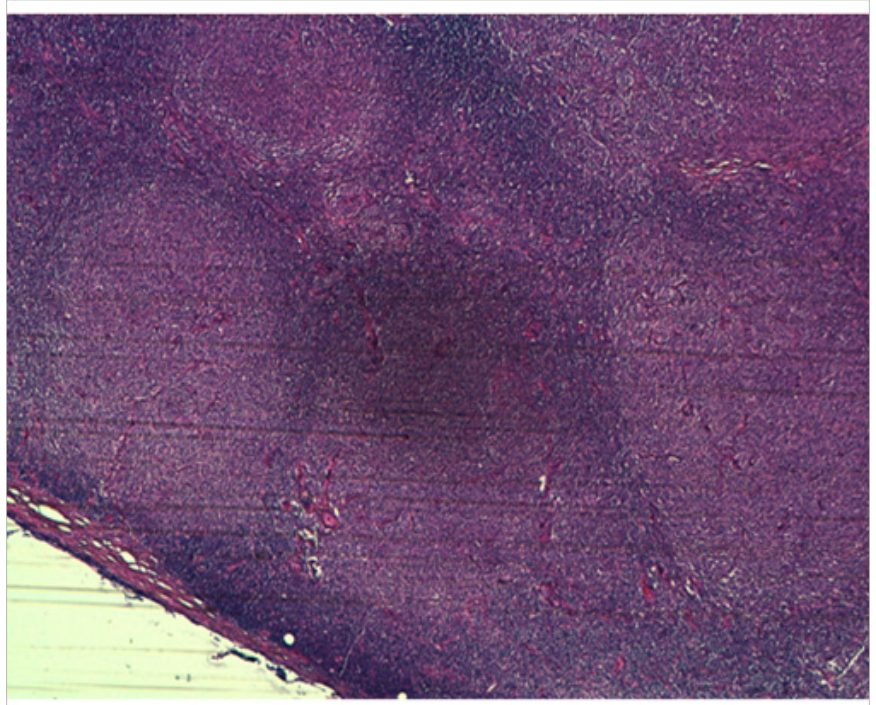

Figure 4 Follicular pattern in inguinal adenopathy. Tumor composed of atypical large lymphoid cells with pleomorphic nuclei and prominent nucleolus.

\section{Discussion}

The risk of lymphoma in patients with IBD is mainly associated with the use of immunosuppressive drugs (such as thiopurines or antiTNF.) In our case, the rapid evolution (Colonoscopy a year ago with minimal aphthae in the rectum and sigma, not very compatible with the Inflammatory well-known with little activity) and being only in treatment with mesalazine at sub-therapeutic doses (1.2gr) and with some cycle of isolated steroids, not being associated to any of the expected drugs. 


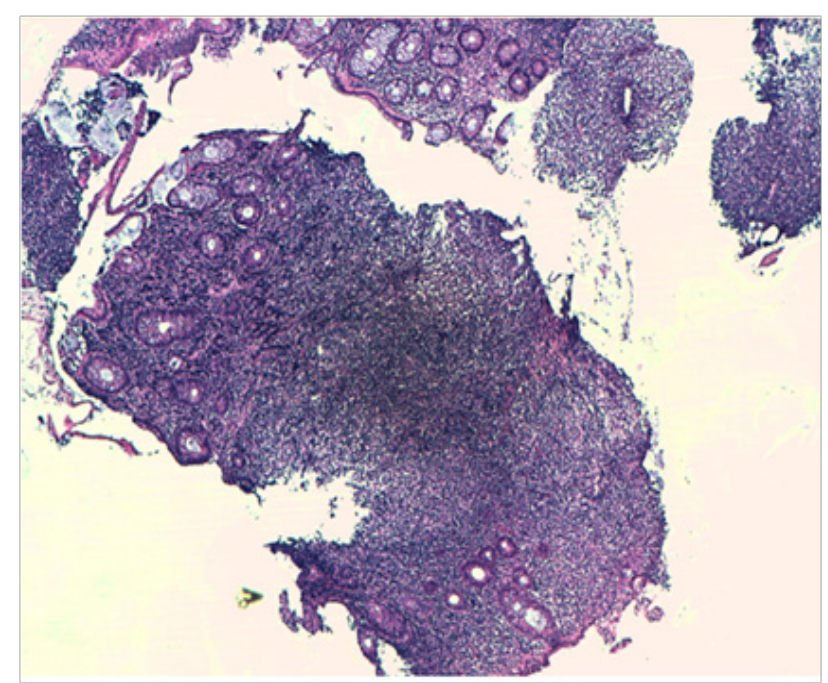

Figure 5 Follicular pattern in colon sample.

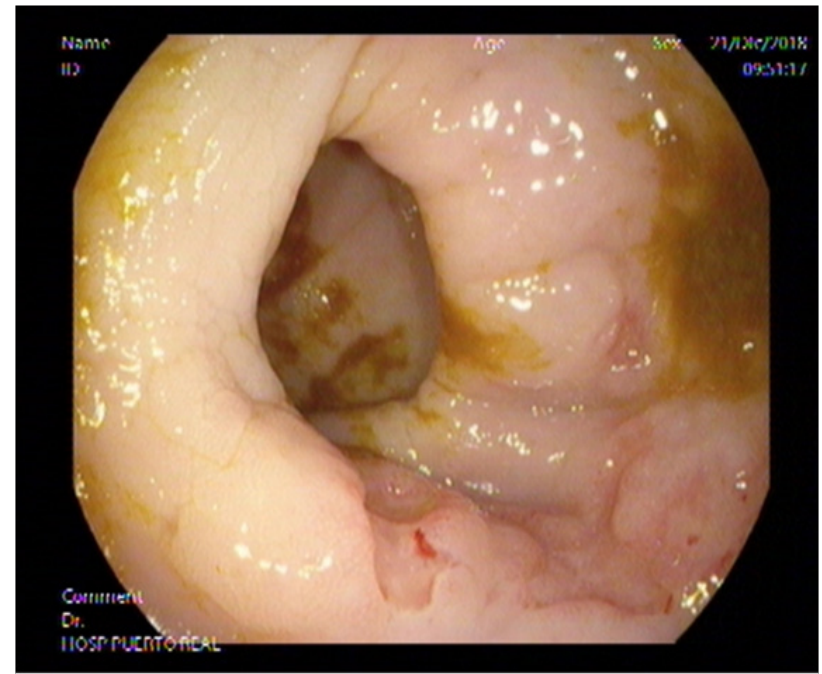

Figure 6 Diffuse non-Hodgkin lymphoma of large B cells of rectum in a patient with a history of ulcerative colitis on colonoscopic examination. (Prechemotherapy injury).

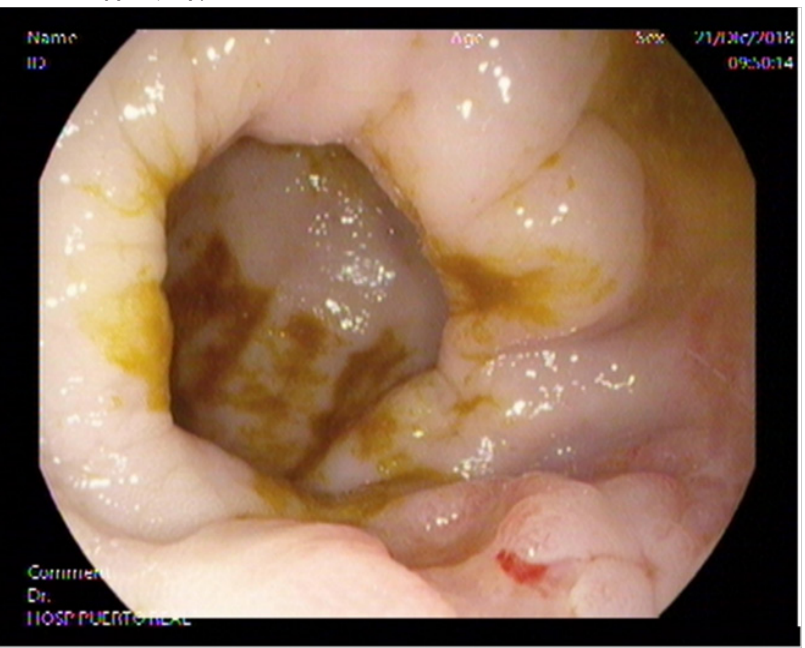

Figure 7 Diffuse non-Hodgkin lymphoma of large B cells of rectum in a patient with a history of ulcerative colitis on colonoscopic examination. (prechemotherapy injury).

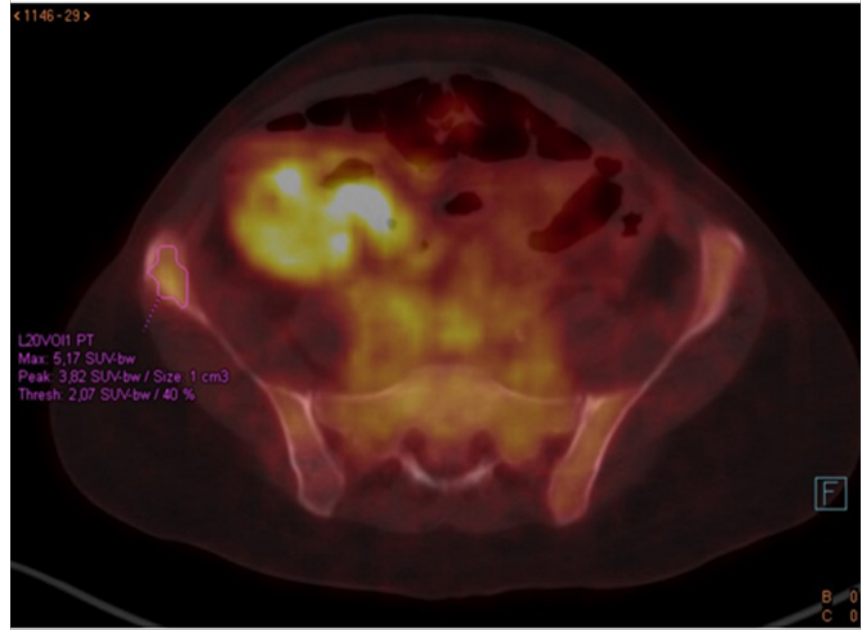

Figure 8 Bone uptake by PET.

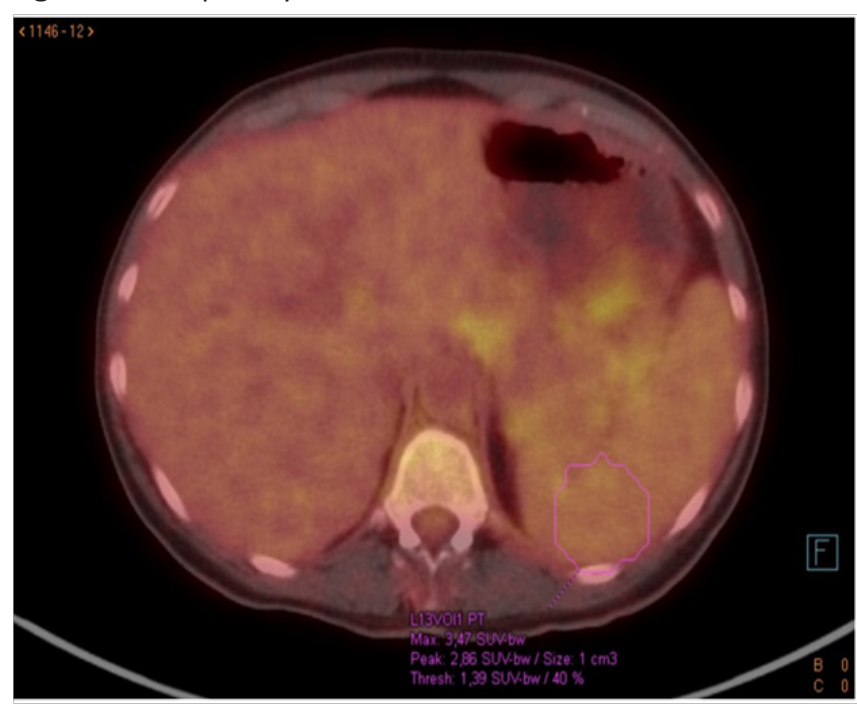

Figure 9 Splenic uptake by PET.

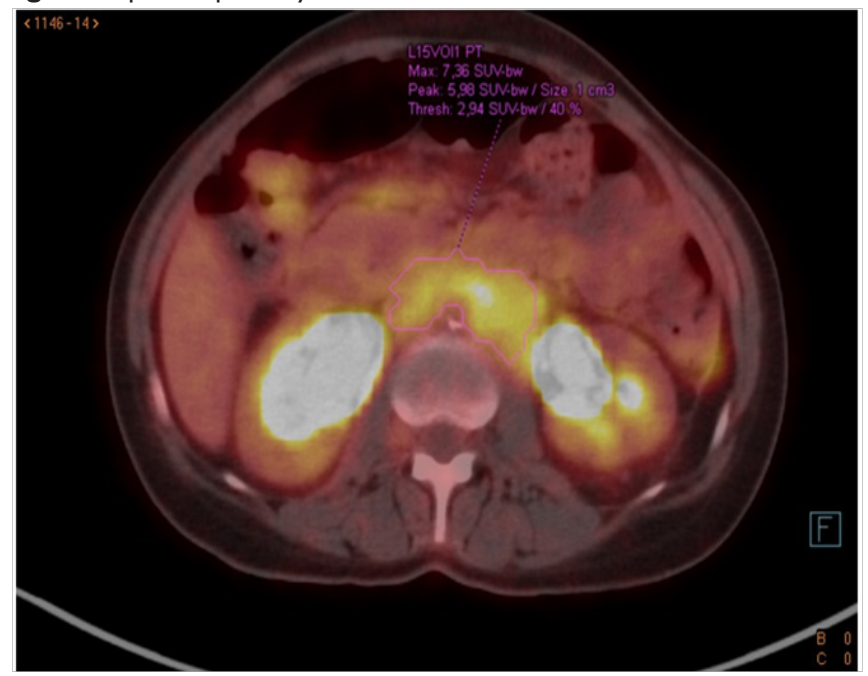

Figure 10 Retroperitoneal uptake by PET.

Given the form of presentation, the differential diagnosis was a challenge due to the unusual way of debut and finally after imaging 
tests and histopathological and immunohistochemical techniques, the referred diagnosis was reached, finding the patient stable at the present time and in remission.

Primary rectal lymphoma is infrequent, constituting only $0.05 \%$ of all primary neoplasms of the rectum. ${ }^{17}$ Its frequency is less than primary lymphoma of ascending colon and cecum, given the greater amount of lymphoid tissue in that area compared to the rest of the colon and rectum. ${ }^{7}$ The clinical, iconographic and endoscopic presentation are nonspecific and subtle, so diagnosis is difficult, being most of the time indistinguishable from other colon diseases, either neoplastic or inflammatory as it occur in our case.

When lymphoma is located in the rectum, the main clinical features are rectal bleeding, proctalgia, tenesmus, and changes in the intestinal habit (diarrhea or constipation). ${ }^{18}$ In some cases, the diagnosis is made incidentally, as it was presented in our patient. ${ }^{19}$

Although the majority of primary colorectal lymphomas are nonHodgkin B-cell lymphomas, studies in China and Korea have shown a higher percentage of T-cell lymphoma, which would have a worse prognosis. ${ }^{20,21}$ PET/CT are techniques of choice for the staging and extension of the evaluation of the presence of a given mass, area of stenosis and ganglionic involvement. ${ }^{22}$ Colonoscopy may show a variable involvement of the mucosa, whether it is a mass, an ulceration or infiltration ${ }^{20}$ and allows obtaining biopsies for a pathological diagnosis.

At present time the risk of lymphoma has become a relevant variable to take into consideration when setting the therapeutic strategy in patients with IBD. Persistent chronic inflammation may play a role in the promotion of carcinogenesis and the development of lymphoma precursor cells, which would lead to overstimulation and continuous proliferation of the immune system..$^{23}$ However, unlike what happens with rheumatoid arthritis 15 , the incidence of LGIP in patients with IBD is relatively low, indicating that IBD alone would not be a definitive independent risk factor in the development of lymphoma. ${ }^{24}$

Infection by EBV is another known risk factor associated with the appearance of a lymphoma; Dayshard et al. showed that those patients with IBD treated with thiopurine who later developed a lymphoma had more frequent EBV in the tumor cells, ${ }^{25}$ which we could not demonstrate in our patient after examining minutely the biopsy samples.

Finally, the use of thiopurinics and anti-TNF has also been associated with the risk of lymphoma, although evidence is scarce. In the case of thiopurinics, studies are contradictory, probably due to bias in the population studied and the duration of therapy. ${ }^{10,11,26,27}$ Studies have suggested a 4-year threshold for thiopurine therapy as a risk factor for developing a lymphoma, ${ }^{28,29}$ a situation that did not occur in our patient. The risk of lymphoma associated with the use of anti-TNF in patients with IBD is difficult to quantify, since most patients have treatment associated with thiopurine or methotrexate concomitantly ${ }^{30}$ Recently, a large population of a multicenter study of over 11,011 patients followed up for a median of 98 months showed that neither immunosuppressants nor anti-TNF drugs therapy seem to increase the risk of developing a lymphoma or other extracolonic cancer. ${ }^{31}$

In summary, managing colorectal lymphoma requires a multidisciplinary evaluation, where prognosis and survival of the patient is determined by the stage of the lymphoma at the moment of diagnosis. Regarding treatment alternatives, there is no consensus on the best therapeutic strategy, with the options being: surgery, chemotherapy and radiotherapy, either as a single or combined treatment. ${ }^{14,32-34}$ In our patient, chemotherapy with an R-CHOP scheme was chosen, achieving criteria of complete remission after 6 cycles, without complications. In relation to the effect of chemotherapy on the evolution of IBD, different studies have shown its beneficial effect. ${ }^{35,36}$ The evolution of our patient has been favorable, being evaluated three months after the end of his chemotherapy, being presently in clinical remission (clinical May index 0) and endoscopic (Mayo endoscopic index 1 at the level of rectum and sigmoid.

\section{Conclusion}

In conclusion, although LGIP is infrequent entity, it should be considered in patients with IBD who do not show a favorable or torpid evolution. The involvement of IBD and the immunological bases that promote lymphocyte activation, together with the role played by thiopurine and anti-TNF treatments in the appearance of lymphoma remains controversial and must be elucidated.

\section{Acknowledgments}

None.

\section{Conflicts of interest}

Author declares their are no conflicts of interest.

\section{References}

1. Quera R, Flores L, Simian D, et al. Rectal diffuse large B cell lymphoma appearing after immunosuppression for ulcerative colitis. Report of one case. Rev Med Chil. 2017;145(10):1342-1348.

2. Freeman C, Berg JW, Cutler SJ. Occurrence and prognosis of extranodal lymphomas. Cancer. 1972;29():252-260.

3. Koch P, del Valle F, Berdel WE, et al. Primary gastrointestinal nonHodking's lymphoma:I. Anatomic and histologic distribution, clinical features, and survival data of 371 patients registered in the German Multicenter Study GITHNL 01/92. J Clin Oncol. 2001;19(18):38613873.

4. Gurney KA, Gurney KA, Cartwright RA. Increasing incidence and descriptive epidemiology of extranodal non-Hodgkin lymphoma in parts of England and Wales. Hematol J. 2002;3(2):95-104.

5. Ghimire $\mathrm{P}, \mathrm{Wu}$ GY, Zhu L. Primary gastrointestinal lymphoma. World $J$ Gastroenterol. 2011;17(6):697-707.

6. Arora N, Manipadam MT, Pulimood A, et al. Gastrointestinal lymphomas:pattern of distribution and histological subtypes: 10 years experience in a tertiary centre in South India. Indian J Pathol Microbiol. 2011;54(4):712-719.

7. Ge Z, Liu Z, Hu X. Anatomic distribution, clinical features, and survival data of 87 cases primary gastrointestinal lymphoma. World J Surg Oncol. 2016;14:85.

8. Wong MT, Eu KW. Primary colorectal lymphomas. Colorectal Dis. 2006;8(7):586-591

9. Zighelboim J, Larson MV. Primary colonic lymphoma. Clinical presentation, histopathologic features, and outcome with combination chemotherapy. J Clin Gastroenterol. 1994;18(4):291-297.

10. Beaugerie L, Brousse N, Bouvier AM, et al. Lymphoproliferative disorders in patients receiving thiopurines for inflammatory bowel disease:a prospective observational cohort study. Lancet. 2009;374(9701):16171625 . 
11. Kandiel A, Fraser AG, Korelitz B, et al. Increased risk of lymphoma among inflammatory bowel disease patients treated with azathioprine and 6-mercaptopurine. Gut. 2005;54(8):1121-1125.

12. Siegel CA, Marden SM, Persing SM, et al. Risk of lymphoma associated with combination anti-tumor necrosis factor and immunomodulator therapy for the treatment of Crohn's disease:a meta-analysis. Clin Gastroenterol Hepatol. 2009;7(8):874-8781.

13. Cai S, Cannizzo F Jr, Bullard Dunn KM, et al. The role of surgical intervention in non-Hodgkin's lymphoma of the colon and rectum. Am J Surg. 2007;193(3):409-412.

14. Tevlin R, Larkin JO, Hyland JM, et al. Primary colorectal lymphoma - a single centre experience. Surgeon. 2015;13(3):151-155.

15. Baecklund E, Iliadou A, Askling J, et al. Association of chronic inflammation, not its treatment, with increased lymphoma risk in rheumatoid arthritis. Arth Reum. 2006;54(3):692-701.

16. Smedby KE, Baecklund E, Askling J. Malignant lymphomas in autoimmunity and inflammation:a review of risks, risk factors, and lymphoma characteristics. Cancer Epidemiol Biomarkers Prev. 2006;15(11):2069-2077.

17. Dood GD. Lymphoma of the hollow abdominal viscera. Radiol Clin North Am. 1990;28(4):771-783.

18. Quayle FJ, Lowney JK. Colorectal lymphoma. Clin Colon Rectal Surg. 2006;19(2):49-53.

19. Shepherd NA, Hall PA, Coates PJ, et al. Primary malignant lymphoma of the colon and rectum. A histopathological and immunohistochemical analysis of 45 cases with clinicopathological correlations. Histopathology. $1988 ; 12(3): 235-252$.

20. Wang MH, Wong JM, Lien HC, et al. Colonoscopic manifestations of primary colorectal lymphoma. Endoscopy. 2001;33(7):605-609.

21. Kim YH, Lee JH, Yang SK, et al. Primary colon lymphoma in Korea:a KASID (Korean association for the study of intestinal disease) study. Dig Dis Sci. 2005;50(12):2243-2247.

22. Lee HJ, Han JK, Kim TK, et al. Primary colorectal lymphoma:spectrum of imaging findings with pathologic correlation. Eur Radiol. 2002;12(9):2242-2249.

23. Copie-Bergman C, Niedobitek G, Mangham DC, et al. Epstein-Barr virus in B-cell lymphomas associated with chronic suppurative inflammation. J Pathol. 1997;183(3):287-292.

24. Watanabe N, Sugimoto N, Matsushita A, Maeda A, Nagai K, Hanioka K, et al. Association of intestinal malignant lymphoma and ulcerative colitis. Intern Med. 2003;42(12):1183-1187.
25. Dayharsh GA, Loftus EV Jr, Sandborn WJ, et al. Epstein-Barr viruspositive lymphoma in patients with inflammatory bowel disease treated with azathioprine or 6-mercaptopurine. Gastroenterology. 2002;122(1):72-77.

26. Armstrong RG, West J, Card TR. Risk of cancer in inflammatory bowel disease treated with azathioprine:a UK population-based case-control study. Am J Gastroenterol. 2010;105(7):1604-1609.

27. Lakatos PL, Lovasz BD, David G, et al. The risk of lymphoma in patients with inflammatory bowel diseases:results from a population-based cohort in Eastern Europe. J Crohn Colitis. 2013;7(5):385-391.

28. Khan N, Abbas AM, Lichtenstein GR, et al. Risk of lymphoma in patients with ulcerative colitis treated with thiopurines:a nationwide retrospective cohort study. Gastroenterology. 2013;145(5):1007-1015.

29. Beigel F, Steinborn A, Schnitzler F, et al. Risk of malignancies in patients with inflammatory bowel disease treated with thiopurines or anti-TNF alpha antibodies. Pharmacoepidemiol Drug Saf. 2014;23(7):735-744.

30. Lichtenstein GR, Feagan BG, Chen RD, et al. Drug therapies and the risk of malignancy in Crohn's disease:Results from the TREATTM registry. Am J Gastroenterol. 2014;109(2):212-223.

31. Chaparro M, Ramas M, Benítez JM, et al. Extracolonic cancer in inflammatory Bowel Disease:Data from the GETTECCU Eneida Registry. Am J Gastroenterology. 2017;112(7):1135-1143.

32. Tang TC, Kuo MC, Chang H, et al. Primary colonic lymphoma:an analysis of 74 cases with localized large-cell lymphoma. Eur J Haematol. 2011;87(1):28-36.

33. Kim SJ, Kang HJ, Kim JS, et al. Comparison of treatment strategies for patients with intestinal diffuse large B-cell lymphoma:surgical resection followed by chemotherapy versus chemotherapy alone. Blood. 2011;117(6):1958-1965.

34. Jeong JH, Kim S, Yoon DH, et al. Clinical characteristics, treatment, and outcome of primary rectal lymphoma:a single center experience of 16 patients. Blood Res. 2017;52(2):125-129.

35. Barta Z, Tóth L, Zeher M. Pulse cyclosphosphamide therapy for Inflammatory Bowel Disease. World J Gastroenterol. 2006;12(8):12781280 .

36. Axelrad JE, Fowler SA, Friedman S, Ananthakrishnan A, Yajnik V. Effects of cancer treatment on Inflammatory Bowel Disease. Clin Gastroenterol Hepatol. 2012;10(9):1021-1027. 